\title{
Communication Styles in Collaborative Peer Learning around Interactive Table
}

\author{
Izdihar Jamil, Sriram Subramanian \\ Interaction \& Graphics Group \\ University of Bristol, UK \\ \{jamil,sriram\}@cs.bris.ac.uk
}

\begin{abstract}
Despite technological, interaction and co-located collaboration advances on multi-touch interactive tables there is little or no knowledge of how people communicate around these surfaces. Communication is important as it acts as a tool for humans to express themselves. Through communication we are able to share information, ideas and emotions, make decisions and build relationships with people. Communication is a key aspect in our everyday life and it is therefore important to understand how we communicate around technology. My work examines the verbal and non-verbal aspects of communication when a group of people collaborate with each other around interactive tables. Findings from my research will provide the community with a deep and systematic analysis of how communication plays a role in the use interactive tables. This will lead to a repertoire of tools for designing and deploying applications on interactive tables that will better support collaboration through positive and meaningful communication. In this paper I will detail my experiences in dealing with video data and analysis.
\end{abstract}

Interactive multi-touch tabletops. Children. Collaborative learning. Communication.

\section{INTRODUCTION}

The characteristics of interactive tables inherited from traditional tabletop workspaces (noninteractive) and coupled with digital information promote a fitting environment for small group collaboration (Preece et al., 2007). The applications of those tables are seen in various areas such as medicine (Piper and Hollan, 2008), conflict management (Stock et al., 2008), entertainment (Jordà et al., 2007) and education (Jamil et al., 2011). With advances in interactive tabletop technology and increased levels of affordability in recent years, there has been a growing interest in exploring their use within educational contexts. Central to this interest are arguments relating to demonstrated benefits of peer collaboration in children's learning and has become an increasing aspect of children's educational experience (Webb and Palincsar, 1996).

The aim of such experiences is to allow children to jointly explore particular areas, discussing and exchanging ideas and perspective and thereby facilitating their understanding. Key to the success of these peer collaborations from a learning perspective is the extent to which children participate in the collaboration (Rogoff, 1990). In particular it is the extent to which peers talk and the nature of this talk that comprises this participation that is argued to be key to successful learning
(Teasley, 1995). For example, successful collaborative learning has been demonstrated to happen when accompanying talk contains more explanations (Teasley, 1995).

Building on these findings, this work further explores the behavior of children as they collaborate around digital tabletops. The motivation behind this is based on Dourish's (Dourish, 2004) idea of "interactional model of context"- where the main concern of context here is how do people: 1) accomplish and sustain a shared understanding of the context of their action? 2) develop and evolve their practices as they interact with each other? Context is not something that is defined or determined. Rather it is an outcome or accomplishment that arises out of the interaction and that outcome is considered meaningful to participants. Based on the work by Sacks on 'being ordinary' (Sacks, 1984), context is something that is generated in the moment (i.e. all the time) that moves people into taking actions and performing activities consistent with their context.

Although there has been some recent work in the literature looking at similar studies, they mainly elaborates on pragmatics rather than understanding the underlying context. For example in their work Pinelle et al. (Pinelle et al., 2009) mainly focuses on the interaction techniques on the tabletop rather than understanding the social 
interaction that occurs between participants above and around the tabletop whilst using particular tabletop techniques. Furthermore, studies of tabletops in real-world settings with ecologically appropriate tasks are still quite rare.

By studying this topic, I aim to:

1. Produce a research framework to systematically analyse communication styles between different interaction techniques in three different countries, namely the UK, India and Finland.

2. Produce a holistic coding scheme and analysis process based on existing literature to investigate the communication styles.

3. Produce applications that foster learning and group communication that are ecologically appropriate tasks suitable for real-world settings.

4. Produce a guide for designers when using different interaction techniques to produce collaborative learning tasks for children.

In exploring this area, we will be better able to understand the impact of interaction techniques on communication during collaborative peer learning when using interactive tables. In this paper, I will present three experiments that I have conducted. In each, I will briefly explain the following points:

1. An overview of the experiments

2. Video data collection and analysis

3. Data presentation

\section{EXPERIMENT 1}

This study presents the findings of a user study investigating conversational patterns across three conditions of table-based interaction (direct touch interactive table, pantograph interactive table and non-digital table) for different types of educational activities (Nacenta et al., 2007). In this study, we had 28 students, aged between 11-13 years performing two collaborative learning activities (spider diagram and classification) with different tabletop interaction techniques and compare the effects of these techniques on patterns of conversational utterances (Jamil et al., 2011). In particular, we compare interactive tables using pantograph and direct touch techniques (as used in Nacenta et al. (Nacenta et al., 2007)) with an equivalent non-digital table where the objects are physical and moveable. In exploring the effects of these interaction techniques on utterance patterns, we will be able to understand better their impact on collaborative peer learning. See Fig. $1 \mathrm{a}$ and $1 \mathrm{~b}$.

\subsection{Video data collection and analysis}

Approximately two hours of video data was collected. Conversations from the video were transcribed and separated into natural units of speech: utterances (Aronoff and Rees-Miller, 2001). Drawing on utterance categories from work in group communication and communication in peer based learning (e.g. (Harris et al., 2009, Rogers et al., 2009, (Harris et al., 2009, Rogers et al., 2009), we coded the utterances in the transcripts according to 8 different conversation categories that would best reflect the peer learning conversation for our study:

(1) Group identification (GI) - utterances addressing the whole group or use of plural pronouns (we, us) that identify members as part of a group.

(2) Interdependence (INT) - utterances related to the need for teamwork and collaboration.

(3) Directives (DIR) - commands, requests as a means to get a listener to perform an action.

(4) Social organisation (SO) - utterances that involved planning, managing, organising and coordinating group activities.

(5) Tasks (TSK) - utterances related to the activities (classification or spider diagram) and topic (for example Energy and Animals) such as relationships between concepts.

(6) Reflection (RFL) - utterances related to broader discussion of the learning topic.

(7) Interaction methods (IM) - utterances related to methods of accessing and manipulating objects.

(8) Playfulness (PLY) - utterances that relate to playing and fooling around.

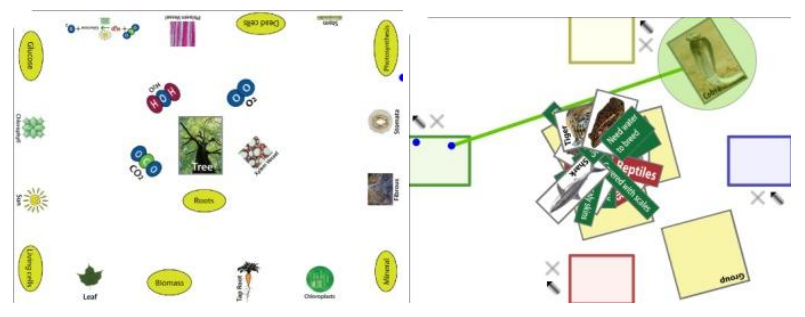

Fig. 1a (left). Spider diagram using a direct touch technique. Fig. 1b (right). Classification using a pantograph technique. The blue dots on the green pantograph represent the user's fingers. For a group of 6 , an additional two pantographs were added on each horizontal side of the tabletop.

Once the coding was complete, we then calculated the utterances per minute (upm) for each utterance category in the three tabletop interaction conditions and two task conditions. Measuring this in terms of "upm" is a well-established approach from the existent literature (Harris et al., 2009, Rogers et al., 2009). "upm" allows us to examine the form of conversation taking place regardless of the time taken to complete the tasks. It therefore allowed us to compare utterance types between the different conditions in a way that a simple measure of total utterances would not, allowing us to compensate for variations in task completion times. 


\subsection{Data presentation}

To explore the effects of interaction techniques further, univariate ANOVA was used to compare upm for all the different utterance categories. For those utterance categories where there was a significant effect we conducted further pairwise comparisons (with Bonferroni adjustment for multiple comparisons) to establish where the differences between interaction techniques lay. We discuss these comparisons alongside qualitative data to facilitate the interpretation of these findings.

\section{EXPERIMENT 2}

We present a study of children in two local schools in Delhi, India engaged in a peer-learning task around interactive table. In the study, we explore how the school children exhibit particular types of collaboration strategies and touch input techniques when dealing with digital objects. In particular, we highlight a number of behaviours of interest, such as how the children would move the same digital object on the table together. We also discuss how the children work in close proximity to each other and dynamically organize their spatial positions in order to work together, as well as establish territory and control. We go on to examine some of the finger-based interaction and manipulation strategies that arise in these contexts. Further information can be found in (Jamil et al., 2012).

\subsection{Video data collection and analysis}

We recruited 139 pupils (aged between 11 and 13 years old) consisting of 110 females and 29 males from local two schools in Delhi, India (Jamil et al., 2011). The study lasted for three days. The participants were divided into 27 groups of 4 to 6 pupils. The tasks performed were similar to Experiment 1. During the session, children were exposed to both interaction techniques and learning activities. After each session we would switch the order of the conditions randomly. Video was used to record the physical and verbal behaviour of all the groups performing the activities. Our analysis draws on a detailed examination of the video recordings and focuses on the collaborative achievement of the tasks. We articulate the interaction details of how gestures, talk and action are produced, coordinated, made visible and understood with respect to the table and on screen objects (Jamil et al., 2012).

\subsection{Data presentation}

We used evidence-based interpretation to illustrate our findings and observations following the methodology of existing literature on interaction analysis (Jordan and Henderson, 1995). The forms of evidence used include sequence of video frames or vignettes depicting the children's interaction with digital objects. In this example, we first describe the interplay that occurred ( $T a b$ 1) between the children whilst interacting on the multi-touch tabletop. This interplay is supported with images (Fig. 1a and 1b) to illustrate the interaction. We then critically analysed the interaction to further understand our findings before deducing the interaction.
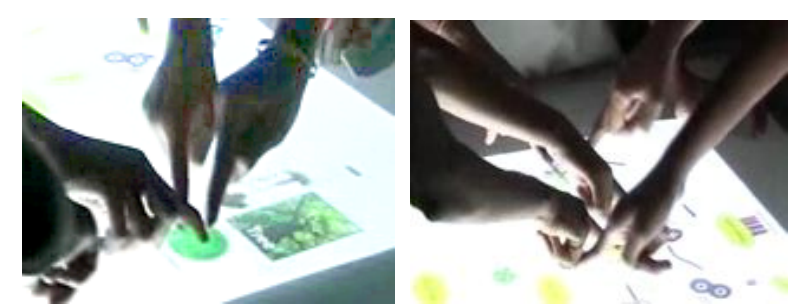

Figure 1a (left). Three students touching and moving chloroplasts towards tree simultaneously. Figure 1b

(right). All five members of Group 11 were seen working within close proximity of each other

Table 1: Children interacting with each other in organising the relationships between tree and chloroplasts.

\begin{tabular}{|c|c|c|}
\hline $28: 56$ & $\begin{array}{l}\text { P5:"Chloropl } \\
\text { asts should } \\
\text { go from here } \\
\text { to here!" }\end{array}$ & $\begin{array}{l}\text { P5 pointed to chloroplasts } \\
\text { (located in the middle of the } \\
\text { tabletop), then moved her arm } \\
\text { and pointed to tree (located at the } \\
\text { right side of the table). P5 worked } \\
\text { on another object. }\end{array}$ \\
\hline 29:00 & $\begin{array}{l}\text { P3:"Move } \\
\text { this!" }\end{array}$ & $\begin{array}{l}\text { P3 touched chloroplasts while } \\
\text { glancing at P4. }\end{array}$ \\
\hline & & \\
\hline
\end{tabular}

\section{EXPERIMENT 3}

This study presents an observational study of children in United Kingdom, India and Finland collaborative around the multi-touch tabletops using the direct touch technique. The purpose of this study is to observe how children communicate and interact with each other whilst socially organizing each other around multi-touch tabletops.

\subsection{Video data collection and analysis}

Video was used to record the physical and verbal behaviour of all the groups performing the activities. At the moment, I am analyzing the data using similar methods as Experiment 2. Our analysis draws on a detailed examination of the video recordings and focuses on the collaborative achievement of the tasks (Jamil et al., 2012).

\subsection{Data presentation}

In terms of data presentation, I intend to follow similar format in Experiment 2 when articulating the interaction details of how gestures, talk and action are produced, coordinated, made visible and understood with respect to the table and on screen objects. I plan to use evidence-based interpretation 
to illustrate our findings and observations following the methodology of existing literature on interaction analysis (Jordan and Henderson, 1995). The forms of evidence used include sequence of video frames or vignettes depicting the children's interaction with digital objects.

\subsection{Difficulties in analyzing the data}

At the moment I am experiencing difficulties in analysing the data due to the following points:

- Each study is conducted with a slight variation from each other due to fulfilling its respective objectives. The data set itself is very rich, therefore would it be possible for me to 'filter' certain elements of each study to create a congruent analysis?

- What would be the most acceptable method in dealing with cross-cultural data?

- What would be the best guidelines or methodology in looking at similarities and differences between cross-cultural data?

- Finally, in which area of research methodologies where all three of my studies can congruently and reasonable be accepted?

\section{CONCLUSION}

The classroom is a challenging environment for evaluation, thus now we believe that our current and future findings in deducing the communication patterns of collaborative peer learning tasks can be a valuable asset towards understanding how the next generation of how $\mathrm{HCl}$ will impact education in the future not only in the UK, but also in other continents. There are challenges remain when dealing with cross-cultural data. It is my intention that in this workshop I will be able to address the above concerns in ensuring the data is dealt without compromising its integrity.

\section{REFERENCES}

Aronoff, M. \& Rees-Miller, J. 2001. The handbook of linguistic, Blackwell Handbooks in Linguistics. Oxford:Blackwell.

Dourish, P. 2004. What we talk about when we talk about context. Personal and Ubiquitous Computing, 8, 19-30.

Harris, A., Rick, J., Bonnett, V., Yuill, N., Fleck, R., Marshall, P. \& Rogers, Y. Year. Around the table: are multiple-touch surfaces better than single-touch for children's collaborative interactions? In: Proc. of CSCL, 2009 Rhodes, Greece. 1600104, 335344.

Jamil, I., O'Hara, K., Perry, M., Karnik, A. \& Subramanian, S. Year. The Effects of Interaction
Techniques on Talk Patterns in Collaborative Peer Learning around Interactive Tables. In: Proc. of CHI, 2011. ACM, 3043-3052.

Jamil, I., Perry, M., O'Hara, K., Karnik, A., Marshall, M. T., Jha, S., Gupta, S. \& Subramanian, S. Year. Group Interaction on Interactive Multi-touch Tables by Children in India. In: Proc. of IDC, 2012. ACM. Jorda, S., Geiger, G., Alonso, M. \& Kaltenbrunner, M. Year. The reacTable: exploring the synergy between live music performance and tabletop tangible interfaces. In: Proc. of TEI, 2007 Baton Rouge, Louisiana. 1226998: ACM, 139-146.

Jordan, B. \& Henderson, A. 1995. Interaction Analysis: Foundations and Practice. The Journal of Learning Sciences, 4, 39-103.

Nacenta, M. A., Pinelle, D., Stuckel, D. \& Gutwin, C. Year. The effects of interaction technique on coordination in tabletop groupware. In: Proc. of GI, 2007 Montreal, Canada. 1268550: ACM Press, 191-198.

Pinelle, D., Barjawi, M., Nacenta, M. \& Mandryk, R. Year. An evaluation of coordination techniques for protecting objects and territories in tabletop groupware. In: Proc. of $\mathrm{CHI}, 2009$ Boston, MA, USA. 1519025: ACM, 2129-2138.

Piper, A. M. \& Holland, J. D. Year. Supporting medical conversations between deaf and hearing individuals with tabletop displays. In: Proc. of CSCW, 2008 San Diego, CA, USA. ACM Press, 147-156.

Preece, J., Rogers, Y. \& Sharp, H. 2007. Interaction Design: Beyond Human-Computer Interaction, Wiley.

Rogers, Y., Lim, Y.-K., Hazelwood, W. \& Marshall, P. 2009. Equal Opportunities: Do Shareable Interfaces Promote More Group Participation Than Single User Displays? Human-Computer Interaction, 24, 79-116.

Rogoff, B. 1990. Apprentinces in thinking: Cognitive development in a Social Context, New York: Simon \& Schuster.

Sacks, H. 1984. On doing 'being ordinary'. In: Atkinson and Heritaghes (eds) Structures of Social Action, Cambrdige University Press, New York.

Stock, O., Zanacaro, M., Koren, C., Rocchi, C., Eisikovits, Z., Goren-Bar, D., Tomasini, D. \& Weiss, P. Year. A co-located interface for narration to support reconciliation in a conflict: initial results from Jewish and Palestinian youth. In: Proc. of $\mathrm{CHI}$ EA, 2008 Florence, Italy. ACM, 1583-1592.

Teasley, S. 1995. The Role of Talk in Children's Peer Collaborations. Developmental Psychology, 31.

Webb, N. \& Palincsar, A. 1996. Group processes in the classroom, In D.C. Berliner \& R.C Calfee (Eds), Handbook of educational psychology. New York: Simon \& Schuster. 